\title{
Nonlinear Constrained Optimization by a Nonrandom Complex Method
}

\author{
R. A. Mitchell and J. L. Kaplan ${ }^{1}$ \\ Institute for Basic Standards, National Bureau of Standards, Washington, D.C. 20234
}

(September 4, 1968)

\begin{abstract}
A nonrandom complex method is described for application to optimization problems characterized by nonlinear objective and constraint functions involving continuous and/or discrete optimization variables. The method is a mutation of the "complex" method (involving a pseudo-random process) developed by M. J. Box. Application of the method is demonstrated by two minimum weight structural analysis problems: (1) an $n$-degree elliptical elastic ring with sinusoidally varying cross section dimensions; and (2) a rib-stiffened, simply supported elastic plate. The ring problem has six continuous independent variables, and the plate problem has four independent variables, two of which are discrete.
\end{abstract}

Key Words: Curved bar analysis; discrete variables; elastic; force transducer; minimum weight; $n$-degree elliptical ring; nonlinear programming; nonrandom complex method; optimization; rib-stiffened plate; structurā analysis.

\section{Introduction}

There are many engineering problems which have an unlimited number of adequate solutions, but which have an optimum solution with regard to some criterion such as weight or cost. This paper describes a nonrandom complex method for seeking the optimum solution for a class of such problems characterized by nonlinear objective and constraint functions of continuous and/or discrete variables. The nonrandom complex method is a mutation of the "complex" method of Box [1]. ${ }^{2}$ Both the nonrandom method and the Box method have an important advantage over many other methods for nonlinear optimization in that they do not require computation of partial derivatives of the functions.

The nonrandom method and the Box method begin a search by generating a configuration of points (an initial complex) whose coordinates are values of the independent optimization variables. At least one point of the initial complex (the initial point) must be known, in advance, to fall inside all constraint bounds of the problem. All other points of the complex are computed to fall inside or on the constraint bounds.

In the Box method, the points of an initial complex are generated by a pseudo-random process. Numerical results by Hilleary [2], using the Box method, demonstrate that the rate of convergence to a solution is dependent on the character of the initial complex, and

${ }^{1}$ Present address: Mathematics Department, University of Maryland, College Park, Maryland.

${ }^{2}$ Figures in brackets indicate the literature references at the end of this paper. that a poor initial complex may result in failure of the method to converge.

In the nonrandom complex method reported here, the character of the initial complex is influenced by the initial point to a much greater degree than in the Box method. Thus, a good initial point leads to a relatively good initial complex. This characteristic is used to advantage by generating a new initial complex about a new initial point if the convergence of a search sequence becomes excessively slow or stops. The same characteristic also facilitates a more efficient discrete variable optimization than would be expected from the Box method. The nonrandom method imposes a lower bound on initial complex size by selecting initial complex points in such a way that at least one-half the constraint bounded range of each independent variable (in the one-dimensional subspace containing the initial point) is included within the complex span.

The nonrandom complex method has been used successfully for the following two minimum weight structural analysis problems:

1. An $n$-degree elliptical elastic ring with sinusoidally varying cross-section dimensions, loaded by two forces acting in opposite directions along a diameter; and

2. A rib-stiffened simply supported plate, loaded by in-plane compressive forces.

The ring problem has six continuous independent variables, and the plate problem has four independent variables, two of which are discrete. Both problems have nonlinear objective and constraint functions. A large class of optimization problems fall within the range of mathematical complexity represented by these two problems. 


\section{List of Symbols}

$x_{k}$ independent variable of the optimization problem,

$g_{i}$ implicit constraint function,

$h_{i}$ implicit constraint lower bound,

$k_{i}$ implicit constraint upper bound,

$E^{m} \quad m$-dimensional Euclidean space,

$P_{j}$ a point of the optimization complex,

$a_{k}$ lower explicit bound on $x_{k}$,

$b_{k} \quad$ upper explicit bound on $x_{k}$,

$\alpha$ reflection factor of complex optimization method,

$m$ the number of independent variables of the optimization problem,

$p$ the number of independent discrete variables of the optimization problem,

$L$ total force applied to ring,

$P=L / 2$, force applied to one quadrant of ring,

$\delta$ curved bar deflection, one-half ring deflection,

$r, \theta$ polar coordinates of ellipse,

$n$ degree of ellipse,

$a, b$ semimajor and semiminor axes of ellipse,

$h$ ring cross-section width,

$t$ ring cross-section thickness normal to tangent of ellipse,

$c$ average of maximum and minimum ring width,

$d$ average of maximum and minimum ring thickness,

$e$ dimensionless parameter of ring width variation,

$f$ dimensionless parameter of ring thickness variation,

$s$ arc length variable measured along ellipse,

$M$ moment,

$N$ normal force,

$V$ shearing force,

$k$ curvature of ellipse,

$g$ integral defined in equations (7) and (8),

$\nu$ Poisson's ratio,

$E$ modulus of elasticity,

$\sigma$ normal stress,

$S$ design maximum stress,

$\lambda$ scale factor,

$W$ weight,

$\gamma$ material density,

$N_{l}$ normal force per unit width of plate,

$l, w$ length and width of rib-stiffened plate,

$t_{p} \quad$ flat plate thickness,

$t_{r}$ rib thickness,

$b_{p}$ rib spacing,

$b_{r}$ rib width,

$H=t_{p}+b_{r}$, total thickness of rib-stiffened plate.

\section{The Nonrandom Complex Method}

The following paragraphs describe the nonrandom complex method as it is applied to a continuous variable problem. Adaptation of the method for discrete variable optimization is described later.

The nonrandom complex method searches for a set of values of the independent continuous variables $x_{1}, x_{2}, \ldots ., x_{m}$ which gives a minimum value of an arbitrary objective function $f\left(x_{1}, x_{2}, \ldots ., x_{m}\right)$, subject to explicit constraints on the variables, and subject to implicit constraint bounds on arbitrary functions of the variables. The implicit constraint functions $g_{i}\left(x_{1}, x_{2}, \ldots ., x_{m}\right)$ are subject to inequalities of the form $h_{i} \leqslant g_{i} \leqslant k_{i}$, where the bounds $h_{i}$ and $k_{i}$ represent arbitrary limits and either one or both may appear.

To begin the method it is required that a point $\left(x_{1}^{\prime}, x_{2}^{\prime}, \ldots ., x_{m}^{\prime}\right)$, in $m$-dimensional Euclidean space, $E^{m}$, be known which is feasible (i.e., does not violate any constraint) and which does not lie on any constraint bound. Using this initial point, a set of $2 m+1$ points in $E^{m}$ is then generated by the following procedure:

1. Denote the $j$ th point in the set of $2 m+1$ points by $P_{j}$. Let $P_{1}=\left(x_{1}^{\prime}, x_{2}^{\prime}, \ldots ., x_{m}^{\prime}\right)$.

2. Denote the explicit constraints on the independent variables by $a_{k} \leqslant x_{k} \leqslant b_{k}$ for $k=1,2,$. . ., $m$. Set $k=1$.

3. Let $P_{2 k}=\left(x_{1}^{\prime}, x_{2}^{\prime}, \ldots ., x_{k-1}^{\prime}, b_{k}, x_{k+1}^{\prime}, \ldots ., x_{m}^{\prime}\right)$. This point is the same as the initial point $P_{1}$ except in the $k$ th coordinate, where $x_{k}^{\prime}$ has been replaced by its explicit upper bound $b_{k}$.

4. Evaluate all implicit constraint functions at $P_{2 k}$. If all are satisfied, move on to step 5. If any implicit constraint is violated, set

$$
P_{2 k}=\left(x_{1}^{\prime}, x_{2}^{\prime}, \ldots, x_{k-1}^{\prime}, \frac{b_{k}+x_{k}^{\prime}}{2}, x_{k+1}^{\prime}, \ldots, x_{m}^{\prime}\right)
$$

This moves $P_{2 k}$ one-half the distance toward the initial point $P_{1}$. Once again, evaluate the implicit constraint functions at $P_{2 k}$, go to step 5 if they are satisfied, or move $P_{2 k}$ one-half the remaining distance to $P_{1}$ otherwise. Repeat the process until $P_{2 k}$ is feasible.

5. Let $P_{2 k+1}=\left(x_{1}^{\prime}, x_{2}^{\prime}, \ldots ., x_{k-1}^{\prime}, a_{k}, x_{k+1}^{\prime}, \ldots ., x_{m}^{\prime}\right)$. This point is the same as the initial point $P_{1}$ except in the $k$ th coordinate, where $x_{k}^{\prime}$ has been replaced by its explicit lower bound $a_{k}$.

6. Proceed with point $P_{2 k+1}$ as in step 4 , moving $P_{2 k+1}$ one-half the distance to the initial point $P_{1}$ until $P_{2 k+1}$ is feasible.

7. Reiterate steps 3 through 6 for $k=2,3, \ldots, m$. This gives a collection of $2 m+1$ points $\left\{P_{1}, P_{2}, \ldots\right.$., $\left.P_{2 m+1}\right\}$ which is the initial complex.

The method proceeds to search for an optimum by repeatedly altering the complex, one point at a time, as follows. The objective function $f\left(x_{1}, x_{2}, \ldots, x_{m}\right)$ is evaluated at each complex point $P_{j}$. The complex point with the worst (greatest) objective function value is selected, and the centroid of the remaining $2 m$ points is computed. The worst complex point is then discarded and replaced by a new point, with the exception that the second worst point is discarded if the worst point is also the newest complex point. That is, a given conplex point is not permitted to be changed in consecutive iteration cycles. A provisional replacement point is computed which is:

1. Collinear with the discarded (worst or second worst) point and the centroid of the $2 m$ complex points excluding the worst point; 
2. On the side of the centroid opposite the discarded point; and

3. Located $\alpha$ times as far from the centroid as was the discarded point, where $\alpha$ is a constant parameter called the reflection factor.

If the provisional replacement point violates an explicit constraint on any variable, the corresponding coordinate is set equal to the violated constraint bound. If the new provisional point then violates an implicit constraint, it is repeatedly replaced by a new point, located one-half the distance to the centroid of the $2 m$ points, until a feasible new complex point is found. The new centroid of all $2 m+1$ complex points is then determined, and the objective function at that centroidal point is evaluated and used as an index of convergence. This completes one cycle of the iterative search process.

During a search, a series of cycles may yield an oscillating value of the centroidal objective function with little or no net convergence. If a preassigned number of consecutive oscillating cycles yield no net improvement in the centroidal objective function value, a new initial complex is generated, using the best centroid yet computed as the initial point.

A search is stopped if a preassigned number of consecutive cycles yield no change in the value of the centroidal objective function greater than a preassigned small amount. This condition may indicate that an approximation of the global optimum has been found. But, the same condition could indicate that a relative (nonglobal) optimum has been found, or that the complex has collapsed to a small size due to the reflection factor being too small or due to some other characteristic of the particular problem. Cycle count and elapsed time stops are also used to prevent excessive computer time.

A search may also be stopped if the centroid of the $2 m$ nonworst complex points lies on or outside a constraint bound. This can be caused by a nonconvex constraint surface or, if the complex is small, it can be caused by numerical rounding errors.

If a search is stopped before a satisfactory approximate optimum is found, it can be restarted by generating a new initial complex, using one of the better complex centroids already computed as the initial point. A centroid somewhat offset inside all constraint bounds, and not necessarily the centroid with the lowest objective function value, is used for this purpose.

\section{Discrete Variable Optimization}

If variables $x_{1}$ through $x_{p}$ of the $m$ optimization variables, where $p \leqslant m$, are limited to known sets of discrete values, the following adaptation of the nonrandom complex procedure is applicable. All $m$ optimization variables are temporarily assumed to be continuous and an optimum continuous variable solution $\bar{x}=\left(\bar{x}_{1}, \bar{x}_{2}, \ldots, \bar{x}_{m}\right)$ is found by the nonrandom complex method. A set of $4^{p}$ neighboring dis- crete value points is then generated by the following procedure:

1. Select the two discrete values of the $k$ th discrete variable, where $k \leqslant p$, that are greater than $\bar{x}_{k}$ and that most nearly approximate $\bar{x}_{k}$.

2. Select the two discrete values of $x_{k}$ that are less than or equal to $\bar{x}_{k}$ and that most nearly approximate $\bar{x}_{k}$.

3. Reiterate steps 1 and 2 for $k=1,2, \ldots, p$. This gives 4 discrete approximate values for each $\bar{x}_{k}$, $k \leqslant p$.

4. Generate the $4^{p}$ distinct combinations of the discrete values obtained by step 3 . Each combination is a set of the discrete coordinates for a discrete approximation to the continuous variable solution point $\bar{x}=\left(\bar{x}_{1}, \bar{x}_{2}, \ldots ., \bar{x}_{m}\right)$. The remaining coordinates for the $4^{p}$ discrete points that surround and approximate $\bar{x}$ are the continuous variable coordinates $\bar{x}_{k}$, where $k>p$.

The objective and constraint functions are then evaluated at each of the $4^{p}$ discrete value points, and the best (lowest objective function value) and second best feasible points are selected. These two points are used as the initial points for two nonrandom complex optimization searches. For each search, the discrete variables are held constant and the continuous variables are optimized within the subspace defined by the discrete coordinates. The two resulting solutions are then compared. If the better of the two solutions results from the better initial point, that solution is assumed to be the optimum solution to the problem. If the better solution results from the second best initial point, the next (third) best feasible point of the $4^{p}$ discrete points is used as the initial point for a third continuous variable optimization within the discrete coordinate subspace containing the point. The process is repeated similarly until a solution results that is worse than a previous solution.

If the number of independent discrete variables is such that $4^{p}$ discrete value points would be an excessively large number to evaluate, an alternative procedure is advisable. A reasonable compromise would be to generate and evaluate the $2^{p}$ adjacent discrete value points that bound the continuous variable solution $\bar{x}$.

\section{Elastic Ring Problem}

An earlier paper [3] reported a minimum weight structural analysis of an $n$-degree elliptical elastic ring, with sinusoidally varying cross section dimensions, by a sequential grid method. The same elastic ring optimization problem is here used to demonstrate application of the nonrandom complex method to a difficult continuous variable problem.

The objective of the optimization problem is to find the dimensions of the minimum weight ring that would satisfy the arbitrary force capacity, deflection, and dimensional requirements of the load supporting element of a force transducer. The force capacity requirement is satisfied by a scaling procedure. Ring deflection 


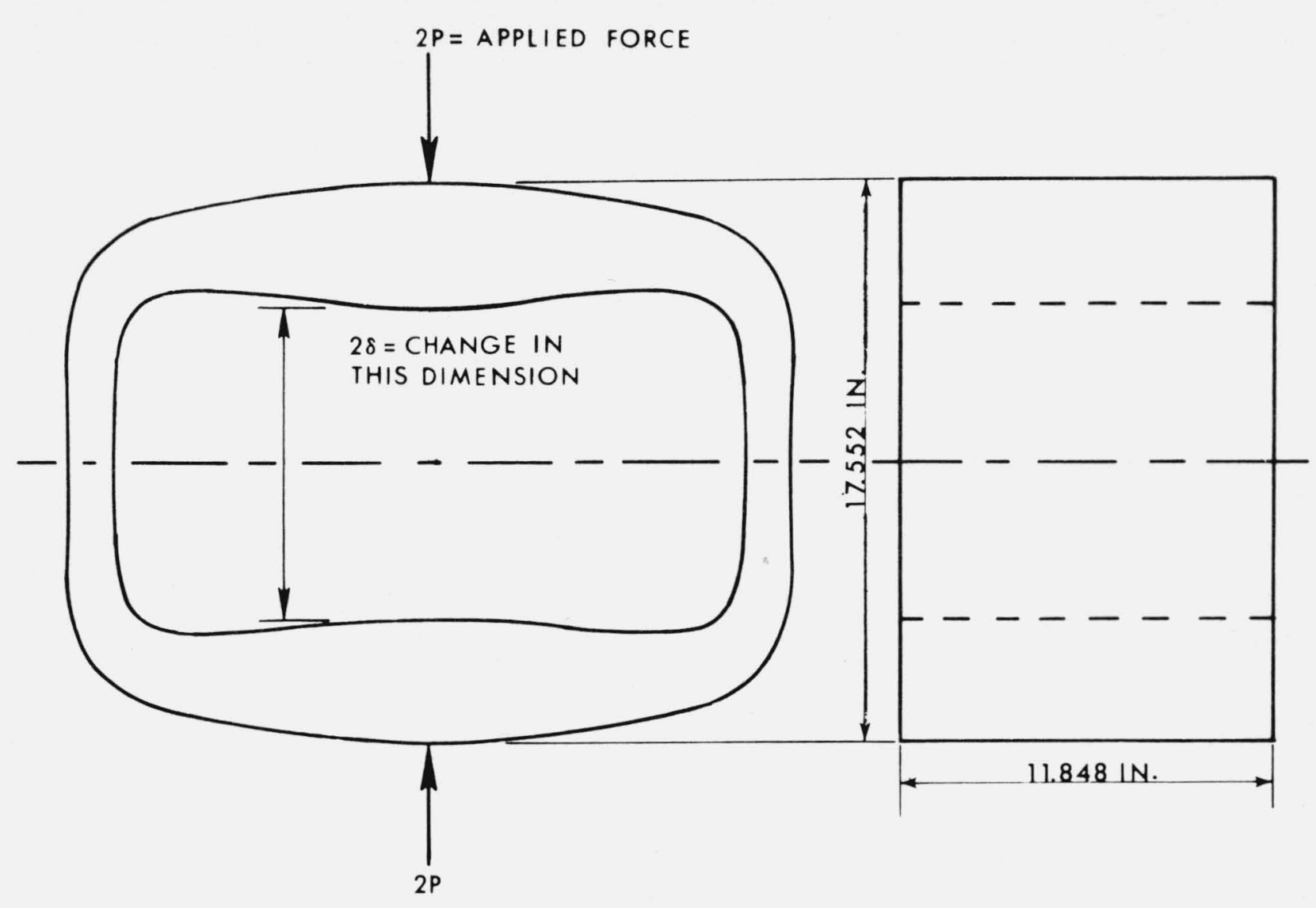

FigURE 1. A 1,000,000 lb capacity n-degree elliptical ring with sinusoidally varying thickness.

and critical ring dimensions are expressed as implicit constraint functions.

An elastic ring force transducer is loaded by two forces acting in opposite directions along a diameter, as shown in figure 1 . Corresponding ring deflections $2 \delta$ are measured and related to applied forces $2 P$ by a calibration factor. A structural analysis (and FORTRAN computer program) for the elastic ring was given in the earlier paper [3]. The ring was analyzed as a thick curved bar, assuming small deflections, plane strain, Winkler-Bach bending stress distribution, uniform normal stress distribution, and parabolic shear stress distribution. The following paragraphs summarize the structural analysis and give the equations required for the nonrandom complex optimization.

For a ring of the shape shown in figures 1 and 2 , the locus of centroids of ring cross sections is an $n$-degree ellipse defined by the equation

$$
r=a b\left(b^{n} \cos ^{n} \theta+a^{n} \sin ^{n} \theta\right)^{-1 / n}, n \geqslant 2 .
$$

Ring rectangular cross-section width $h$ and thickness $t$ are given by

$$
h=c(1-e \cos 2 \theta),
$$

and

$$
t=d(1-f \cos 2 \theta),
$$

in which $c$ and $d$ are positive parameters and $e$ and $f$ are parameters of absolute value less than unity.

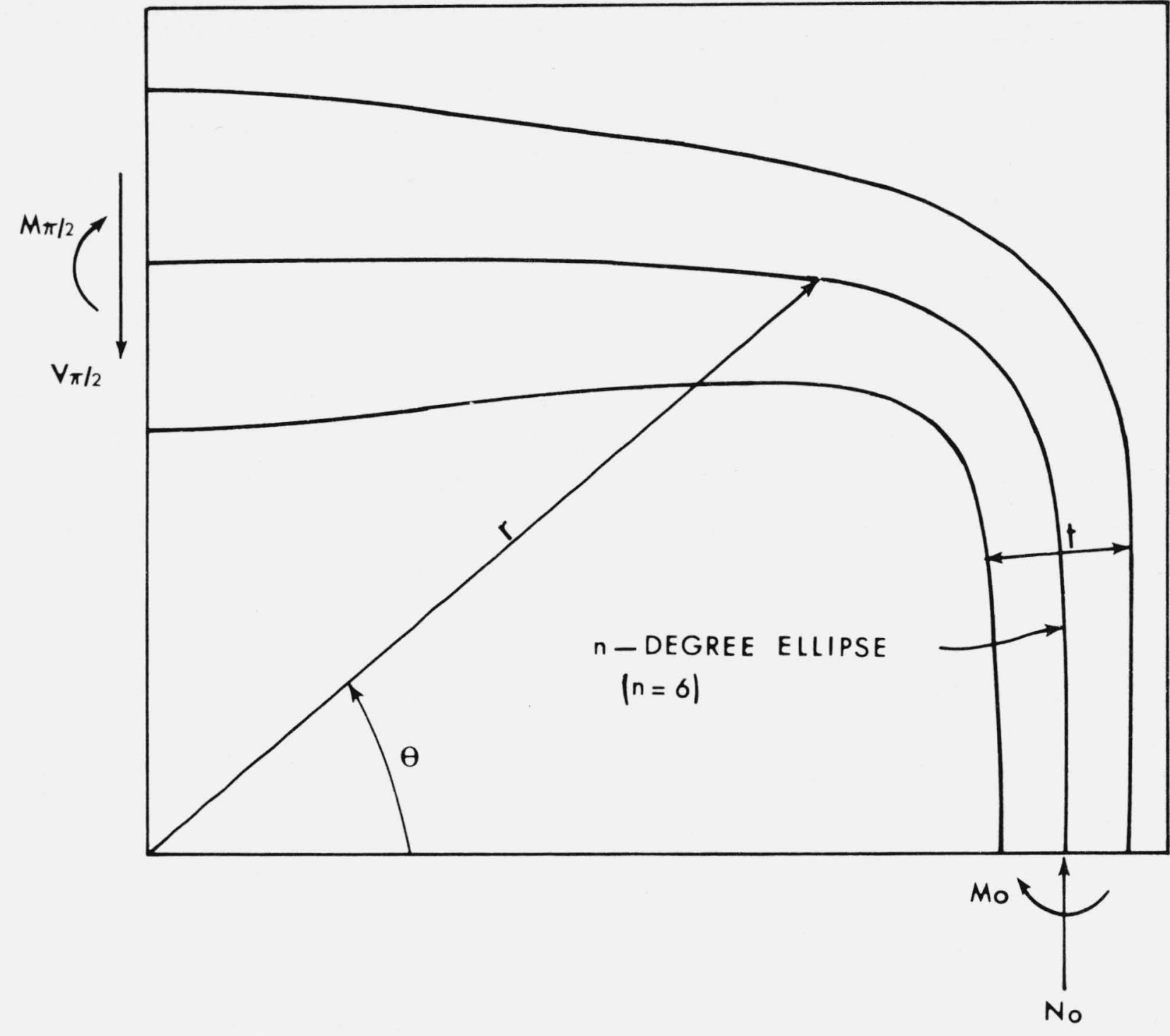

FigURE 2. Loads acting on one quadrant of a ring.

Using these equations, the shape of a ring within this geometric class can be specified by the seven parameters $a, b, c, d, e, f$, and $n$. If $a=b, n=2$, and $e=f=0$, the ring shape is circular with uniform rectangular cross section.

Figure 2 shows the loads acting on one quadrant of the ring. The resultant moment and forces acting on a typical normal cross section are

$$
\begin{gathered}
M=M_{\pi / 2}-P r \cos \theta=P M^{\prime}, \\
N=P\left(\sin \theta \frac{d r}{d s}+r \cos \theta \frac{d \theta}{d s}\right)=P N^{\prime},
\end{gathered}
$$

and

$$
V=P\left(r \sin \theta \frac{d \theta}{d s}-\cos \theta \frac{d r}{d s}\right)=P V^{\prime},
$$

in which $d s$ is the increment of arc length of the $n$ degree ellipse. With the assumed stress distributions, the strain energy of the ring quadrant is obtained by integrating over the quadrant volume. Castigliano's strain energy theorem is used to determine the moment and deflection at the point of load application to be

$$
M_{\pi / 2}=P\left[\frac{\int_{0}^{\pi / 2} \frac{\left(1-\nu^{2}\right) g r \cos \theta}{h} d s-\int_{0}^{\pi / 2} \frac{k \sin \theta}{h t} d r-\int_{0}^{\pi / 2} \frac{k r \cos \theta}{h t} d \theta}{\int_{0}^{\pi / 2} \frac{\left(1-\nu^{2}\right) g}{h} d s}\right],
$$

and

$$
\delta_{\pi / 2}=\frac{P}{E} \int_{0}^{\pi / 2} \frac{1}{h t}\left[N^{\prime 2}+\frac{12}{5}(1+\nu) V^{\prime 2}-\left(1-\nu^{2}\right) g t r \cos \theta M^{\prime}+k M^{\prime} N^{\prime}-k r \cos \theta N^{\prime}\right] d s,
$$


in which

$$
\begin{gathered}
k=\frac{r^{2}+2\left(\frac{d r}{d \theta}\right)^{2}-r \frac{d^{2} r}{d \theta^{2}}}{\left[r^{2}+\left(\frac{d r}{d \theta}\right)^{2}\right]^{3 / 2}}, \\
g=\int_{t}\left[\frac{k}{t}+\frac{y}{(1+k y) \int_{t} \frac{y^{2}}{(1+k y)} d y}\right]^{2} d y,
\end{gathered}
$$

$\nu=$ Poisson's ratio,

$E=$ modulus of elasticity,

and $y$ is the distance from a cross-section centroid to a point in the cross section, positive in the outward direction. The stress in the $d s$ direction on the inner surface of the ring is given by

$\sigma_{T}=P\left[\frac{M^{\prime} k}{h t}-\frac{M^{\prime} t}{h(2-k t) \int_{t} \frac{y^{2}}{(1+k y)} d y}+\frac{N^{\prime}}{h t}\right]$.

For the optimization analysis, it is assumed that the force resisting capacity of the ring is limited by the maximum value of $\sigma_{T}$ for all $\theta$. The possibility of capacity being limited by some other component of stress or combined stress should be checked in any final design analysis.

To make each numerical solution have the required force capacity, all length dimensions in the $r-\theta$ plane are scaled by a factor $\lambda$ that will make the maximum value of $\sigma_{T}$, for the entire ring, equal a prescribed maximum design stress $S$. Cross-section width $h$ is not scaled. The scale factor is

$\lambda=\frac{L_{\lambda}}{2 S}\left[\frac{M^{\prime} k}{h t}-\frac{M^{\prime} t}{h(2-k t) \int_{t} \frac{y^{2}}{(1+k y)} d y}+\frac{N^{\prime}}{h t}\right]_{\text {max. }}$,

in which $S=$ design maximum stress,

$L_{\lambda}=$ required force capacity,

and the maximum value of the bracketed function for all $\theta$ is used.

In the optimization analysis the above equations are first solved for a ring of unit value of mean radius, that is

$$
\frac{a+b}{2}=1
$$

Since $a$ and $b$ are functionally dependent, the ring shape can be specified by the six independent shape parameters $a, c, d, e, f$, and $n$. After the structural equations are solved for the unit size ring (using Simpson numerical integration), the results are scaled to full size using the scale factor $\lambda$. The width parameter $c$ is not scaled during the entire analysis, and the shape parameters $e, f$, and $n$ are nondimensional and are not scaled.

The six shape parameters $a, c, d, e, f$, and $n$ are the six independent variables $x_{1}, x_{2}, \ldots$. ., $x_{6}$, respectively, of the constrained optimization problem. The objec- tive function $f\left(x_{1}, x_{2}, \ldots, x_{6}\right)$ is the scaled ring weight

$$
W=4 \gamma \lambda^{2} \int_{0}^{\pi / 2} h t d s
$$

in which $\gamma$ is the material density. The following six implicit constraint functions $g_{i}$ are applied:

$$
\begin{aligned}
g_{1}= & 2 \lambda \delta_{\pi / 2}=\text { total ring deflection, } \\
g_{2}= & \lambda d(1+|f|)=\text { maximum thickness of ring } \\
& \text { cross section, } \\
g_{3}= & \lambda(2 a+d+d f)=\text { outside width of ring, } \\
g_{4}= & \lambda(4-2 a+d+d f)=\text { outside height of } \\
& \text { ring, } \\
g_{5}= & c(1+|e|)=\text { maximum width of ring cross } \\
& \text { section, }
\end{aligned}
$$

and $g_{6}=\lambda(4-2 a-d-d f)=$ inside clear height of ring.

Implicit lower bounds $h_{i}$ and/or upper bounds $k_{i}$ are imposed on the functions $g_{i}$ to limit ring deflection and dimensions.

The scaling procedure used here could have been avoided by expressing force capacity as an implicit constraint function with a lower bound. It is not known whether such a procedure would be more efficient than the scaling procedure used.

Numerical solutions were obtained for seven different minimum weight elastic ring problems. Tables 1 and 2 give the initial points, the constraint bounds and the solutions obtained. The material properties were: $S=150,000 \mathrm{lb} / \mathrm{in}^{2}, E=30,000,000 \mathrm{lb} / \mathrm{in}^{2}, \nu=0.3$, and $\gamma=0.29 \mathrm{lb} / \mathrm{in}^{3}$. The computations were coded in FORTRAN V and run on a UNIVAC 1108 computer. Time required for a solution ranged from $4 \mathrm{~min}$ to 18 min with a mean of 9 min. The number of initial complex regenerations required for a solution ranged from 2 to 21 with a mean of 9 . A reflection factor of 1.5 was used for all seven solutions.

For each of the two $1,000,000 \mathrm{lb}$ capacity $n$-degree elliptical rings, the best solution reported in the earlier paper [3] (weight $=539.08 \mathrm{lb}$ ) was used as the initial point. For the other five problems, the initial feasible points were obtained by using the relatively simple equations for a thin circular ring of uniform cross section. The thin circular ring equations, although they may be in error by several percent, give a good enough approximation of ring characteristics for beginning an optimization search. The coordinates obtained by the thin ring equations were offset slightly inside their explicit bounds, where that was necessary, to comply with the requirements of the optimization method. To hold a particular parameter constant, the upper and lower explicit bounds were set equal to the initial point coordinate value. 
TABLE 1. Initial points and constraint bounds for elastic ring problems

\begin{tabular}{|c|c|c|c|c|c|c|c|c|c|c|c|c|c|c|c|c|c|c|c|c|c|c|c|c|c|c|}
\hline \multirow{2}{*}{$\begin{array}{c}\text { Force } \\
\text { capacity }\end{array}$} & \multirow{2}{*}{$\begin{array}{c}\text { Problem } \\
\text { dimension }\end{array}$} & \multicolumn{18}{|c|}{ Initial point coordinates and explicit bounds } & \multicolumn{7}{|c|}{ Implicit bounds } \\
\hline & & $a_{1}$ & $a$ & $b_{1}$ & $a_{2}$ & $c$ & $b_{2}$ & $a_{3}$ & $d$ & $b_{3}$ & $a_{4}$ & $e$ & $b_{4}$ & $a_{5}$ & $f$ & $b_{5}$ & $a_{6}$ & $n$ & $b_{6}$ & $h_{1}$ & $k_{1}$ & $k_{2}$ & $k_{3}$ & $k_{4}$ & $k_{5}$ & $h_{6}$ \\
\hline$L b$ & & Inch & Inch & Inch & Inch. & Inch & Inch & Inch & Inch & Inch & & & & & & & & & & Inch & Inch & Inch & Inch & Inch & Inch & Inch \\
\hline 100,000 & 5 & 1.0 & 1.01 & 1.3 & 3.0 & 5.0 & 6.0 & 0.05 & 0.151 & 0.3 & 0.0 & 0.0 & 0.0 & 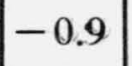 & 0.0 & 0.9 & 2.0 & 2.1 & 20.0 & 0.25 & 0.55 & 4.0 & 40.0 & 40.0 & 6.0 & 9.0 \\
\hline 300,000 & 5 & 1.0 & 1.01 & 1.3 & 4.0 & 8.0 & 10.0 & .1 & 188 & .4 & 0.0 & 0.0 & 0.0 & 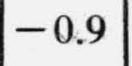 & 0.0 & 0.9 & 2.0 & 2.1 & 20.0 & .25 & .55 & 4.0 & 40.0 & 40.0 & 10.0 & 9.0 \\
\hline $1,000,000$ & 2 & 1.0 & 1.00 & 1.0 & 6.0 & 12.0 & 14.0 & .1 & .250 & .5 & 0.0 & 0.0 & 0.0 & 0.0 & 0.0 & 0.0 & 2.0 & 2.0 & 2.0 & .25 & .55 & 7.0 & 50.0 & 50.0 & 18.0 & 9.0 \\
\hline $1,000,000$ & 5 & 1.0 & 1.21 & 1.3 & 6.0 & 13.3 & 14.0 & .1 & .305 & .5 & 0.0 & 0.0 & 0.0 & $-0.9 \mid$ & 0.5 & 0.9 & 2.0 & 6.0 & 20.0 & .25 & .55 & 7.0 & 50.0 & 50.0 & 18.0 & 9.0 \\
\hline $1,000,000$ & 6 & 1.0 & 1.21 & 1.3 & 6.0 & 13.3 & 14.0 & .1 & .305 & .5 & 0.9 & 0.0 & 0.9 & -0.9 & 0.5 & 0.9 & 2.0 & 6.0 & 20.0 & .25 & .55 & 7.0 & 50.0 & 50.0 & 18.0 & 9.0 \\
\hline $3,000,000$ & 5 & 1.0 & 1.01 & 1.3 & 14.0 & 18.0 & 20.0 & .25 & .311 & .5 & 0.0 & 0.0 & 0.0 & -0.9 & 0.0 & 0.9 & 2.0 & 2.1 & 20.0 & .25 & .55 & 9.0 & 60.0 & 60.0 & 20.0 & 9.0 \\
\hline $9,000,000$ & 5 & 1.0 & 1.01 & 1.3 & 20.0 & 28.0 & 32.0 & .3 & .387 & .5 & 0.0 & 0.0 & 0.0 & -0.9 & 0.0 & 0.9 & 2.0 & 2.1 & 20.0 & .25 & .55 & 15.0 & 85.0 & 85.0 & 32.0 & 9.0 \\
\hline
\end{tabular}

TABLE 2. Solutions for elastic ring problems

\begin{tabular}{|c|c|c|c|c|c|c|c|c|c|c|c|c|c|c|c|c|}
\hline \multirow{2}{*}{$\begin{array}{c}\text { Force } \\
\text { capacity }\end{array}$} & \multirow{2}{*}{$\begin{array}{c}\text { Problem } \\
\text { dimension }\end{array}$} & \multicolumn{6}{|c|}{ Ring shape parameters of unit size ring } & \multicolumn{6}{|c|}{ Implicit constraint function values } & \multirow{2}{*}{$\begin{array}{l}\text { Scale } \\
\text { factor }\end{array}$} & \multirow{2}{*}{$\begin{array}{c}\text { Ring } \\
\text { weight }\end{array}$} & \multirow{2}{*}{ Cycles } \\
\hline & & $a$ & c & $d$ & $e$ & $f$ & $n$ & $g_{1}$ & $g_{2}$ & $g_{3}$ & $g_{4}$ & $g_{5}$ & $g_{6}$ & & & \\
\hline$L b$ & & Inch & Inch & Inch & & & & Inch & Inch & Inch & Inch & Inch & Inch & & $L b$ & \\
\hline 100,000 & 5 & 1.07509 & 5.35807 & 0.14689 & 0.00000 & 0.79775 & 5.36445 & 0.2502 & 1.5078 & 12.4472 & 12.0703 & 5.3581 & 9.0546 & 5.710 & 52.40 & 210 \\
\hline 300,000 & 5 & 1.09246 & 8.40706 & .19659 & .00000 & .67392 & 4.52213 & .2503 & 2.3088 & 15.7788 & 15.0431 & 8.4071 & 10.4256 & 7.016 & 163.17 & 201 \\
\hline $1,000,000$ & 2 & 1.00000 & 12.32346 & .27146 & .00000 & .00000 & 2.00000 & .2501 & 4.1548 & 34.7653 & 34.7653 & 12.3235 & 26.4558 & 15.305 & 1427.90 & 190 \\
\hline $1,000,000$ & 5 & 1.19033 & 11.87379 & .31110 & .00000 & .56132 & 9.39859 & .2509 & 3.8990 & 20.2053 & 16.8975 & 11.8738 & 9.0996 & 8.027 & 492.49 & 179 \\
\hline $1,000,000$ & 6 & 1.19717 & 11.77591 & .31561 & -.02669 & .56049 & 8.15270 & .2512 & 3.9900 & 20.5215 & 16.9984 & 12.0902 & 9.0184 & 8.102 & 497.78 & 263 \\
\hline $3,000,000$ & 5 & 1.02028 & 16.12696 & .33864 & .00000 & .62951 & 5.93016 & .2504 & 6.6805 & 26.2232 & 30.4025 & 16.1270 & 17.0414 & 12.107 & 1686.69 & 522 \\
\hline $9,000,000$ & 5 & 1.02117 & 25.64302 & .45811 & .00000 & .51622 & 5.69466 & .2503 & 9.7050 & 31.6321 & 37.0573 & 25.6430 & 17.6474 & 13.972 & 4819.54 & 414 \\
\hline
\end{tabular}


For the five $n$-degree elliptical rings with uniform cross section width, the ratio of weight to force capacity ranges from $4.92 \times 10^{-4}$ to $5.62 \times 10^{-4}$. At least a rough proportionality between weight and force capacity might be expected for structures of this geometric class. Thin ring equations would predict a constant ratio of weight to force capacity for uniform cross section, circular rings having the same deflection at capacity load.

In each of the seven solutions, ring deflection, $g_{1}$, is near its lower bound, $h_{1}=0.25 \mathrm{in}$. In three of the solutions, inside clear height, $g_{6}$, is near its lower bound, $h_{6}=9.0$ in. Exact minimum weight solutions would apparently lie exactly on at least one of the bounds. Continued iteration would have resulted in closer approximations to exact solutions. But the searches were stopped because of the relatively large computer costs involved and because the convergence rate decreases as an optimum is approached.

The seven solutions include three $1,000,000 \mathrm{lb}$ capacity rings of different geometric complexity. One ring is circular with uniform cross section; one is $n$-degree elliptical with constant cross-section width and sinusoidally varying thickness; and one is $n$-degree elliptical with sinusoidally varying width and thickness. The three cases are respectively two-dimensional, five-dimensional, and six-dimensional optimization problems. All three cases have the same implicit constraint bounds. Both $n$-degree elliptical rings have a weight of about 35 percent of the weight of the circular ring, and the outside dimensions of the more complex rings are significantly less than those of the circular ring. Since the five-dimensional problem is a special case of the six-dimensional problem, an exact solution to the six-dimensional problem would have a weight equal to or less than any solution to the fivedimensional problem. Although both elliptical rings have about the same weight, the ring with nonuniform cross-section width would probably cost more to manufacture than would the other elliptical ring.

\section{Rib-Stiffened Plate Problem}

The minimum weight analysis of a rib-stiffened elastic plate is presented here to demonstrate the application of the nonrandom complex method to a discrete variable problem. It is assumed that the ribstiffened plate is built-up from flat plates that are available in only a limited number of thicknesses.

Figure 3 gives a schematic drawing of a rib-stiffened plate of the type studied. The flat plate is reinforced by a rectangular grid system of ribs having the same dimensions and spacing in both directions. The ribstiffened plate is simply supported along all four edges and loaded only with a compressive load $N_{l}$ in the plane of the plate.

Schmit, Kicher, and Morrow [4] have studied the application of gradient optimization methods to a more general class of rib-stiffened plates, subjected to combinations of both shear and biaxial normal loads. They considered three possible elastic buckling modes to be applicable to their problem. The following specialized forms of the buckling equations used in their study are assumed to apply to the present problem:

1. Critical load for gross buckling of the simply supported, rib-stiffened plate,

$$
N_{c r}=\frac{\pi^{2}}{w^{2}}\left[D_{1}\left(\frac{w^{2}}{l^{2}}+\frac{l^{2}}{w^{2}}\right)+2 D_{3}\right],
$$

in which

$$
\begin{array}{r}
D_{1}=\frac{E H^{3}}{4}\left\{\frac{1}{3\left(1-\nu^{2}\right)}\left(\frac{t_{p}}{H}\right)^{3}+\frac{1}{3}\left(1-\frac{t_{p}}{H}\right)^{3}\left(\frac{t_{r}}{b_{p}}\right)\right. \\
+\frac{\nu}{2\left(1-\nu^{2}\right)}\left(1-\frac{t_{p}}{H}\right)\left(\frac{t_{p}}{H}\right)\left(\frac{t_{r}}{b_{p}}\right)
\end{array}
$$$$
\times\left[\frac{1+\nu}{\left(\frac{\nu}{1-\nu}\right)\left(\frac{t_{p}}{H}\right)+\nu\left(1-\frac{t_{p}}{H}\right)\left(\frac{t_{r}}{b_{p}}\right)}\right.
$$$$
\left.\left.+\frac{1-\nu}{\left(\frac{\nu}{1+\nu}\right)\left(\frac{t_{p}}{H}\right)+\nu\left(1-\frac{t_{p}}{H}\right)\left(\frac{t_{r}}{b_{p}}\right)}\right]\right\},
$$

$$
\begin{aligned}
D_{3}= & \frac{E H^{3}}{4}\left\{\frac{1}{3\left(1-\nu^{2}\right)}\left(\frac{t_{p}}{H}\right)^{3}\right. \\
& \left.+\frac{\left(\frac{\nu}{1-\nu^{2}}\right)\left(\frac{t_{p}}{H}\right)\left(1-\frac{t_{p}}{H}\right)^{2}\left(\frac{t_{r}}{b_{p}}\right)^{2}}{\left[\left(\frac{1}{1-\nu^{2}}\right)\left(\frac{t_{p}}{H}\right)+\left(1-\frac{t_{p}}{H}\right)\left(\frac{t_{r}}{b_{p}}\right)\right]^{2}-\left(\frac{1}{1-\nu^{2}}\right)^{2}\left(\frac{t_{p}}{H}\right)^{2}}\right\},
\end{aligned}
$$

$E=$ modulus of elasticity,

$\nu=$ Poisson's ratio,

and the length dimensions are those indicated in figure 3.

2. Critical load for local buckling of a stiffening rib as a flat plate, simply supported along three edges and free along the fourth edge,

$$
\bar{N}_{c r}=\frac{\pi^{2} E}{12\left(1-\nu^{2}\right)}\left(\frac{t_{r}}{b_{r}}\right)^{2}\left[\frac{b_{p} t_{p}+b_{r} t_{r}}{b_{p}}\right]\left[\left(\frac{b_{r}}{b_{p}-t_{r}}\right)^{2}+0.425\right] \text {. }
$$

3. Critical load for local buckling of a square panel as a flat piate, spanning between two pairs of ribs and simply supported along all four edges,

$$
\tilde{N}_{c r}=\frac{\pi^{2} E t_{p}^{2}}{3\left(1-\nu^{2}\right)\left(b_{p}-t_{r}\right)^{2}}\left[\frac{b_{p} t_{p}+b_{r} t_{r}}{b_{p}}\right] .
$$

The critical material stress in the rib-stiffened plate is assumed to be equal to the applied load divided by the gross cross-sectional area of the flat plate and 

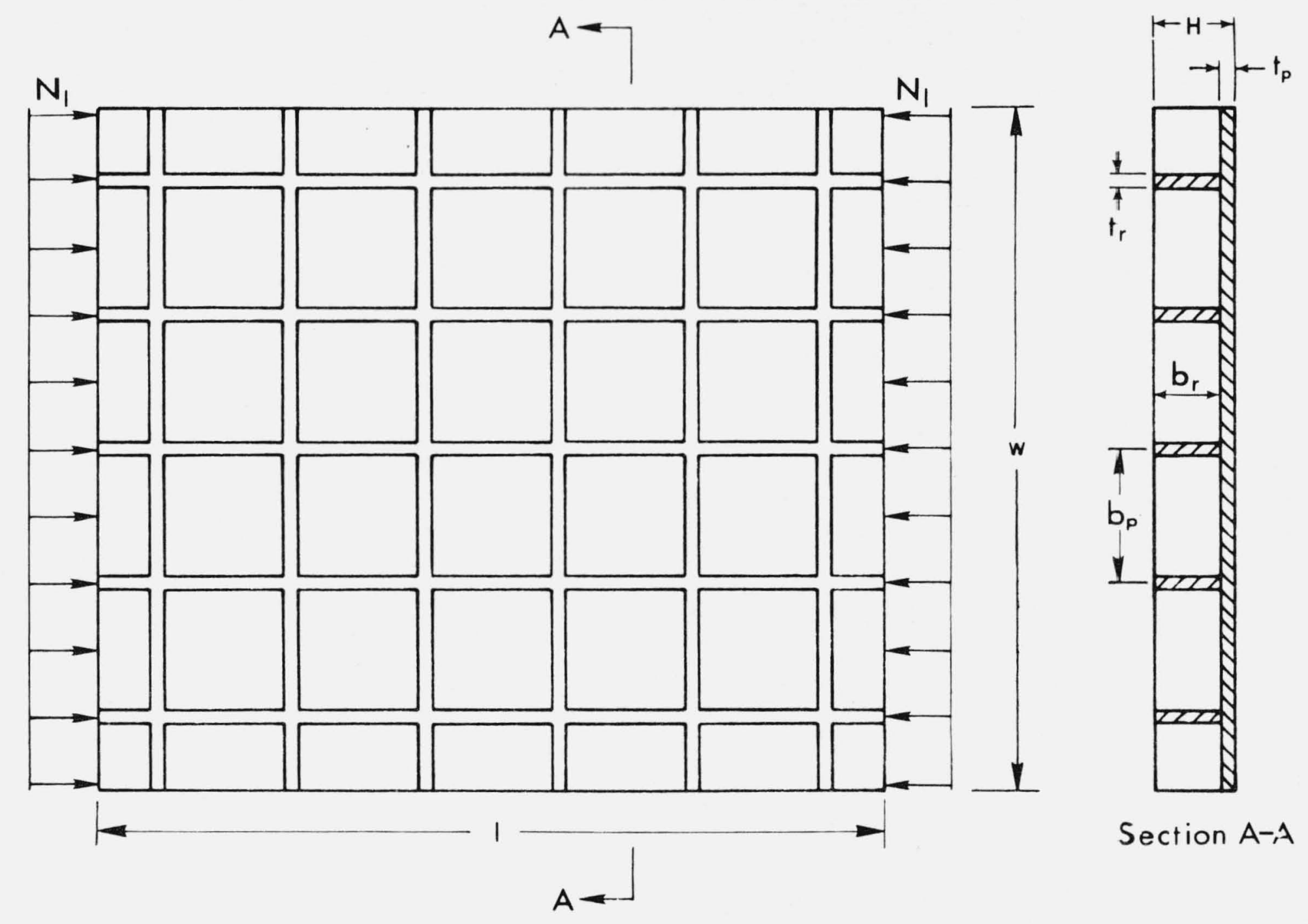

Figure 3. Rib-stiffened plate.

ribs. That is,

$$
\sigma_{l}=\frac{N_{l}}{t_{p}+b_{r}\left(\frac{t_{r}}{b_{p}}\right)} .
$$

The gross weight of the rib-stiffened plate is given approximately by

$$
W=\gamma l w t_{p}\left[1+\frac{b_{r} t_{r}}{b_{p} t_{p}}\left(2-\frac{t_{r}}{b_{p}}\right)\right],
$$

in which $\gamma$ is the material density. Equation (23) does not include the net weight resulting from the fastening of the stiffening ribs to the larger flat plate or to each other.

For the particular numerical problem presented here, plate length and width, $l$ and $w$, are held constant and the four plate dimensions $t_{p}, t_{r}, b_{p}$, and $b_{r}$ are the variable parameters of the optimization search. The plate thicknesses, $t_{p}$ and $t_{r}$, are discrete variables. The objective function is the gross weight of the plate, $W$. The following implicit constraint functions are applied:

$$
\begin{aligned}
& g_{1}=N_{c r}=\text { gross buckling load, } \\
& g_{2}=\bar{N}_{c r}=\text { rib buckling load, } \\
& g_{3}=\tilde{N}_{c r}=\text { panel buckling load, } \\
& g_{4}=\sigma_{l}=\text { critical stress, }
\end{aligned}
$$

$$
\text { and } g_{5}=t_{p}+b_{r}=\text { total stiffened plate thickness. }
$$

The applied load that the plate is required to support is a lower bound on $g_{1}, g_{2}$, and $g_{3}$. The design stress, $S$, is an upper bound on $g_{4}$.
A numerical solution was obtained for a rib-stiffened plate 30 in wide by 40 in long with an applied compressive load $N_{l}=350 \mathrm{lb} /$ in. Lower and upper bounds of 0.5 in and 0.7 in, respectively, were imposed on total stiffened plate thickness, $g_{5}$. Explicit constraint bounds on the variable optimization parameters were as follows (in inches):

$$
\begin{aligned}
& 0.005 \leqslant t_{p} \leqslant 0.500 \\
& 0.005 \leqslant t_{r} \leqslant 0.500 \\
& 2.000 \leqslant b_{p} \leqslant 6.000 \\
& 0.100 \leqslant b_{r} \leqslant 0.700 .
\end{aligned}
$$

The material properties were: $S=20,000 \mathrm{lb} / \mathrm{in}^{2}$, $E=10,500,000 \mathrm{lb} / \mathrm{in}^{2}, \nu=0.32$, and $\gamma=0.101 \mathrm{lb} / \mathrm{in}^{3}$. Forty-four discrete plate thicknesses from 0.0040 in to 0.5800 in were assumed to be available. The computations were coded in FORTRAN $\mathrm{V}$ and run on a UNIVAC 1108 computer. Total computer time required for the solution was $55 \mathrm{~s}$. A reflection factor of 1.6 was used.

It was determined by direct computation that a 0.2043 in thick plate was the thinnest available plate that would support the applied load as a flat plate, without stiffening ribs. Such a plate would weigh about $24.76 \mathrm{lb}$. The 0.2043 in flat plate thickness combined with a reasonable set of rib dimensions were used as the initial point coordinates for the first continuous variable search.

Table 3 gives the initial points and results for the series of five optimization searches employed in finding a discrete variable solution. The result of the first continuous variable search was a solution point near 
TABLE 3. Initial points and results for rib-stiffened plate problem

\begin{tabular}{|c|c|c|c|c|c|c|c|c|c|c|c|}
\hline \multirow{2}{*}{$\begin{array}{l}\text { Optimization } \\
\text { search }\end{array}$} & \multicolumn{4}{|c|}{ Variable parameters } & \multicolumn{5}{|c|}{ Implicit constraint function values } & \multirow{2}{*}{$\begin{array}{l}\text { Gross } \\
\text { weight }\end{array}$} & \multirow[t]{2}{*}{ Cycles } \\
\hline & $t_{p}$ & $t_{r}$ & $b_{p}$ & $b_{r}$ & $N_{c r}$ & $\bar{N}_{e r}$ & $\tilde{N}_{e r}$ & $\sigma_{l}$ & $H$ & & \\
\hline \multirow{3}{*}{$\begin{array}{c}\text { First } \\
\text { continuous }\end{array}$} & $\begin{array}{l}\text { Inch } \\
0.20430\end{array}$ & $\begin{array}{l}\text { Inch } \\
\quad 0.20430\end{array}$ & $\begin{array}{l}\text { Inch } \\
\quad 4.00000\end{array}$ & $\begin{array}{l}\text { Inch } \\
0.30000\end{array}$ & Lb/Inch & Lb/Inch & Lb/Inch & $L b / I n c h^{2}$ & Inch & \multirow[t]{2}{*}{$\begin{array}{l}L b \\
28.3805\end{array}$} & \\
\hline & (Initial & & & & & & & & & & \\
\hline & $\begin{array}{r}.04541 \\
\text { (Res }\end{array}$ & .04526 & 3.44039 & .64625 & 350.00 & 945.27 & 371.19 & 6492.0 & 0.69166 & 7.5510 & 246 \\
\hline \multirow{2}{*}{$\begin{array}{c}\text { Second } \\
\text { continuous }\end{array}$} & $\begin{array}{r}.04767 \\
\text { (Initial }\end{array}$ & t) .05041 & 3.72198 & 63748 & 350.10 & 1242.35 & 365.24 & 6216.3 & .68515 & 7.8563 & \\
\hline & $\begin{array}{r}.03068 \\
\text { (Res }\end{array}$ & .02961 & 2.00111 & .64446 & 350.10 & 350.00 & 374.79 & 8703.1 & .67514 & 6.0128 & 215 \\
\hline \multirow{2}{*}{$\begin{array}{c}\text { Third } \\
\text { continuous }\end{array}$} & $\begin{array}{c}.03068 \\
\text { (Initial }\end{array}$ & t) .02961 & 2.00110 & 64444 & 350.10 & 350.10 & 374.91 & 8702.2 & .67512 & 6.0133 & \\
\hline & $\begin{array}{r}.02989 \\
\text { (Res }\end{array}$ & .02988 & 2.00000 & .64409 & 350.15 & 350.51 & 350.00 & 8858.2 & .67398 & 5.9375 & 111 \\
\hline \multirow{2}{*}{$\begin{array}{c}\text { First } \\
\text { discrete }\end{array}$} & $\begin{array}{l}.03200 \\
\text { (Initial }\end{array}$ & t) .03200 & 2.00000 & .64409 & 376.95 & 430.72 & 430.46 & 8273.2 & .67609 & 6.3565 & \\
\hline & $\begin{array}{r}.03200 \\
\quad \text { Res }\end{array}$ & .03200 & 2.19138 & .64465 & 350.00 & 406.67 & 350.00 & 8451.3 & .67665 & 6.1436 & 91 \\
\hline \multirow{2}{*}{$\begin{array}{l}\text { Second } \\
\text { discrete }\end{array}$} & $\begin{array}{l}.03590 \\
\text { (Initial }\end{array}$ & t) .02850 & 2.00000 & .64409 & 351.38 & 363.79 & 575.24 & 7764.3 & .67999 & 6.5600 & \\
\hline & $\begin{array}{r}.03590 \\
\quad \text { Res } \\
\end{array}$ & .02850 & 2.08008 & .65105 & 350.01 & 350.00 & 528.17 & 7809.0 & 68695 & 6.4985 & 114 \\
\hline
\end{tabular}

the gross buckling constraint and not far from both the panel buckling constraint and a total plate thickness constraint. The continuous variable search was restarted, in a second computer run, using a complex centroid somewhat offset inside all constraints as the initial point. The result of the second continuous variable search was a solution point near a rib spacing constraint, near the gross buckling constraint, near the rib buckling constraint, and not far from the panel buckling constraint. The continuous variable search was again restarted, in a third computer run, and the resulting solution point was near a rib spacing constraint and all three buckling constraints. As a continuation of the third computer run, the two best discrete solution points near the continuous variable solution were selected and used as initial points for two searches within the subspaces defined by the discrete coordinates. The better of the two discrete solutions resulted from the better discrete initial point. Therefore, no additional discrete subspace searches were executed. The gross weight for the discrete solution is about 25 percent of the weight of the thinnest available flat plate that would support the applied load without stiffening ribs. Initial complex regeneration, to overcome excessively slow convergence, was not required during any of the five optimization searches.

\section{Discussion}

Unfortunately, a general method is not available for determining whether or not a solution found by the nonrandom complex method is (approximately) the true optimum. But a careful study of the search record can yield a basis for judgment. The study should include not only the final values (centroidal) of the variables and functions, but also the variation of these values for the entire search. The entire complex, at regular cycle intervals, should also be examined.

Multiple searches along different paths can be used to test apparent optimum solutions. The search path depends on the objective and constraint functions, the constraint bounds, the initial point, the reflection factor, the complex regeneration criterion, and numerical errors. A change in one or more of these factors can cause a search to progress along a different path. Of course the rate of convergence is, in general, different for different search paths.

Discrete variables can occur in practical problems in several different forms. The rib-stiffened plate problem involves two discrete variables with tabular noninteger values. That problem could have been written to include an integer optimization variable by expressing rib spacing as a function of the number of equally spaced longitudinal ribs. Schmit and Mallett [5] have discussed the optimization of material selection by initially treating one material property as an independent variable, with other material properties expressed as continuous functions of the independent material property. With such a procedure, material selection could be treated as a discrete optimization variable in a nonrandom complex search. Similarly, the selection of commercially available elements, such as metal beams and columns, could be optimized by initially expressing element geometric properties as continuous functions of a single independent variable, for example weight per unit length. 
Numerous other optimization methods applicable to problems with nonlinear objective and constraint functions have been discussed in the literature, for example by Box [1], Hilleary [2], Schmit, Kicher, and Morrow [4], Schmit and Mallect [5], and Wilde and Beightler [6]. The limited cornputational experience reported in the present paper is insufficient to warrant a definitive claim of superiority of the nonrandom complex method over other available methods.

\section{Conclusion}

A nonrandom complex method has been developed for application to optimization problems characterized by nonlinear objective and consiraint functions involving continuous and/or discrete optimization variables. The nonrandom method appears to offer significant advantages over the Box [1] method which involves a pseudo-random process. The method was successful in finding approximate minimum weight solutions to:

1. Seven elastic ring structural problems involving from two to six independent variables each; and

2. A rib-stiffened elastic plate structural problem involving four independent variables, two of which are discrete.

These results, for problems of this complexity, suggest that the method is applicable to a large class of optimization problems.
The subroutines for solving the structural equations of the elastic ring problem were coded in FORTRAN V by R. M. Slesser while he was an employee of the National Bureau of Standards Center for Computer Sciences and Technology.

\section{References}

[1] Box, M. J., A new method of constrained optimization and a comparison with other methods, The Computer Journal 8, 42-52 (Apr. 1965).

[2] Hilleary, R. R., The tangent search method of constrained minimization, U.S. Naval Postgraduate School Tech. Rep./Res. Paper No. 59 (Mar. 1966).

[3] Mitchell, R. A., Analysis of $n$-degree elliptical elastic rings of nonuniform cross section, J. Res. NBS 72C (Engr. and Instr.). No. 2, 139-160 (1968).

[4] Schmit, L. A., Jr., Kicher, T. P., and Morrow, W. M., Structural synthesis capability for integrally stiffened waffle plates, J. AIAA 1, No. 12 (Dec. 1963).

[5] Schmit, L. A., Jr., and Mallett, R. H., Structural synthesis and design parameter hierarchy, J. Struct. Div. ASCE 89, No. ST4, (Aug. 1963).

[6] Wilde, D. J., and Beightler, C. S., Foundations of Optimization (Prentice-Hall, Inc., 1967).

(Paper 72C4-282) 